\title{
METABOLISMO LÍQUIDO DA MATÉRIA ORGÂNICA, CALCIFICAÇÃO E FLUXOS DE DIÓXIDO DE CARBONO EM DOIS RECIFES COSTEIROS SUJEITOS A DIFERENTES GRAUS DE IMPACTO: COROA VERMELHA E TAIPUS DE FORA, BAHIA
}

\author{
SOUZA, M.F.L. ${ }^{1}$ \& SANTANA, T.B. ${ }^{2}$ \\ 1. Departamento de Ciências Exatas e Tecnológicas, Universidade Estadual de Santa Cruz, Ilhéus, \\ BA. \\ 2. Universidade Estadual de Santa Cruz - Graduação em Sistemas Aquáticos Tropicais, Ilhéus, BA.
}

\begin{abstract}
Souza, M.F.L. \& Santana, T.B., 2015. Metabolismo líquido da matéria orgânica, calcificação e fluxos de dióxido de carbono em dois recifes costeiros sujeitos a diferentes graus de impacto: Coroa vermelha e Taipus de fora, Bahia. Braz. J. Aquat. Sci. Technol. 19(3). eISSN 1983-9057. DOI: 10.14210/bjast.v19n3. This study is intended to compare physical and chemical variables, net ecosystem metabolism (production - respiration) and calcification-carbonate dissolution rates in two coastal reefs subject to different levels of anthropogenic inputs of nutrients and organic matter. The coast surrounding Coroa Vermelha reef presented a higher degree of urbanization and touristic activities than Taipus de Fora at the time of sampling. Temperature, dissolved inorganic nutrients, total alkalinity, and total suspended solids were significantly higher in Coroa Vermelha reef, probably as a result of the anthropogenic inputs. These variables in Taipus de Fora were comparable to those found in the literature for Recife de Fora, a protected reef farthest from the coast and other less impacted reefs. Total alkalinity, dissolved inorganic carbon and carbon dioxide partial pressure were lower and $\mathrm{pH}$ was higher in the reef flat than at the surrounding seawater. There was a prevalence of an influx of atmospheric carbon dioxide to reef water, net autotrophy and calcification. A significant correlation was observed between net calcification and net community metabolism. The benthic photosynthesis in the reef flat during low tide decreases the carbon dioxide partial pressure and increases the aragonite saturation state, establishing thermodynamic conditions that favor calcification.
\end{abstract}

Keywords: Net autotrophy, Net calcification, Benthic metabolism, Tidal pools, Atmosphere-seawater fluxes.

\section{INTRODUÇÃO}

Os recifes de coral são ecossistemas de elevada biodiversidade que têm despertado grande interesse devido à ocorrência dos processos de calcificação e dissolução de carbonato, fotossíntese e respiração. Estes são os processos que promovem as mudanças no sistema carbonato observadas nestes ecossistemas (Smith, 1973; Gattuso et al., 1993; Gattuso et al., 1999). Estes processos consomem ou liberam carbono inorgânico dissolvido e respondem a mudanças em parâmetros ambientais tais como luz, temperatura (Leclercq et al., 2002) e ao aumento da pressão parcial de dióxido de carbono atmosférico e acidificação dos oceanos (Gattuso et al., 1998; Orr et al., 2005).

O metabolismo líquido da matéria orgânica no ecossistema (MLE) é expresso como a diferença algébrica entre a fotossíntese $(P)$ e a respiração $(R)$. Este metabolismo representa a soma destes processos nas comunidades presentes, mas normalmente a simbiose coral/zooxantelas e as macroalgas são os seus principais contribuintes. Comunidades de microrganismos fotoautotróficos epilíticos e endolíticos também podem estar entre os principais produtores primários em recifes (Tribollet et al., 2006).

Em ecossistemas com autotrofia líquida $(P>R)$ a assimilação de $\mathrm{CO}_{2}$ em excesso da que é produzida pela remineralização da matéria orgânica eleva o $\mathrm{pH}$, a concentração de íons carbonato e o estado de saturação de carbonatos, diminui a concentração de carbono inorgânico dissolvido (CID) e a pressão parcial de $\mathrm{CO}_{2}\left(p \mathrm{CO}_{2}\right)$. A alcalinidade total permanece praticamente inalterada, exceto por pequenas mudanças devido à assimilação de nutrientes. Em ecossistemas com heterotrofia líquida $(P<R)$, ocorrem as alterações contrárias.

O equilíbrio entre as taxas de precipitação e dissolução de carbonatos também é importante para os fluxos de carbono inorgânico. Grande parte da precipitação de carbonato em recifes de coral é promovida por organismos bênticos, principalmente pelos corais escleractíneos. A dissolução normalmente é uma resposta à ação de macro e microperfurantes. Embora pareça paradoxal, a calcificação em recifes de coral libera $\mathrm{CO}_{2}$ na água em uma relação teórica de 1 mol para cada mol de $\mathrm{CaCO}_{3}$ precipitado. Na maior parte dos sistemas marinhos costeiros esta relação real situa-se em cerca de 0,6 moles de $\mathrm{CO}_{2}$ por mol de carbonato precipitado (Frankignoulle et al., 1994; 
Smith, 2013). Isto faz com que recifes de coral com calcificação líquida também apresentem produção líquida de $\mathrm{CO}_{2}$ (Gattuso et al., 1999). O reverso ocorre em ecossistemas com dissolução líquida.

Os fluxos de dióxido de carbono através da interface água-atmosfera também alteram o sistema carbonato em água recifais. A evasão do gás para a atmosfera ou sua dissolução na água vão diminuir ou aumentar respectivamente o pH e a concentração de carbono inorgânico dissolvido, sem alteração na alcalinidade total. Estes fluxos através desta interface são lentos, de forma que em escalas temporais curtas o metabolismo do carbono orgânico e inorgânico (MLE) vai controlar a química do carbonato na água do mar. Desta forma, os fluxos através da interface água-atmosfera em recifes de coral são controlados pelo metabolismo do carbono orgânico ou processos de calcificação e dissolução de carbonato (Falter et al., 2013; Frankignoulle et al., 1994; Frankignoulle and Gattuso 1993; Smith, 1974; Smith, 1981; Ware et al., 1991).

Grande parte dos ecossistemas recifais ao longo da costa do Nordeste apresenta-se como bancos próximos à costa, muitas vezes contínuos à praia ou ligados por um tômbolo arenoso. Esta proximidade os torna sujeitos aos efeitos negativos do crescimento urbano (eutrofização, assoreamento) e do turismo (pisoteamento, remoção de organismos). Em escala regional estes fatores (mudanças globais cumulativas) podem ser mais importantes que as mudanças climáticas globais sistêmicas (Buddemeyer \& Smith, 1999), especialmente em recifes costeiros. Além de constituírem uma ameaça à biodiversidade, estes impactos podem afetar drasticamente o metabolismo dos corais e suas relações ecológicas.

Este estudo visou obter uma primeira caracterização de variáveis físico-químicas e aspectos do metabolismo bêntico do carbono nos recifes costeiros de Coroa Vermelha e Taipus de Fora, situados no litoral sul e Baixo Sul da Bahia, comparando as características físico-químicas, processos metabólicos líquidos (produção-respiração) e de calcificação-dissolução de carbonato. Estes recifes se encontravam em regiões de diferentes graus de urbanização, fluxo turístico e aporte antropogênico de nutrientes e matéria orgânica por ocasião do estudo.

\section{MATERIAL E MÉTODOS}

\section{Área de estudo}

Foram realizadas duas campanhas de amostragem nos recifes de Coroa Vermelha (CV; Santa Cruz de Cabrália, Bahia, 16 19' 56" S, 39 00' $05^{\prime \prime} \mathrm{W}$ ) e Taipus de Fora (TF, Maraú, Bahia, $13^{\circ} 56^{\prime}$ $24^{\prime \prime}$ S, 38 $55^{\prime} 36^{\prime \prime}$ W) em novembro/dezembro de 2004 (Fig. 1). A área de estudo está situada em região de clima tropical úmido, com maré semidiurna, em uma região de mesomarés.

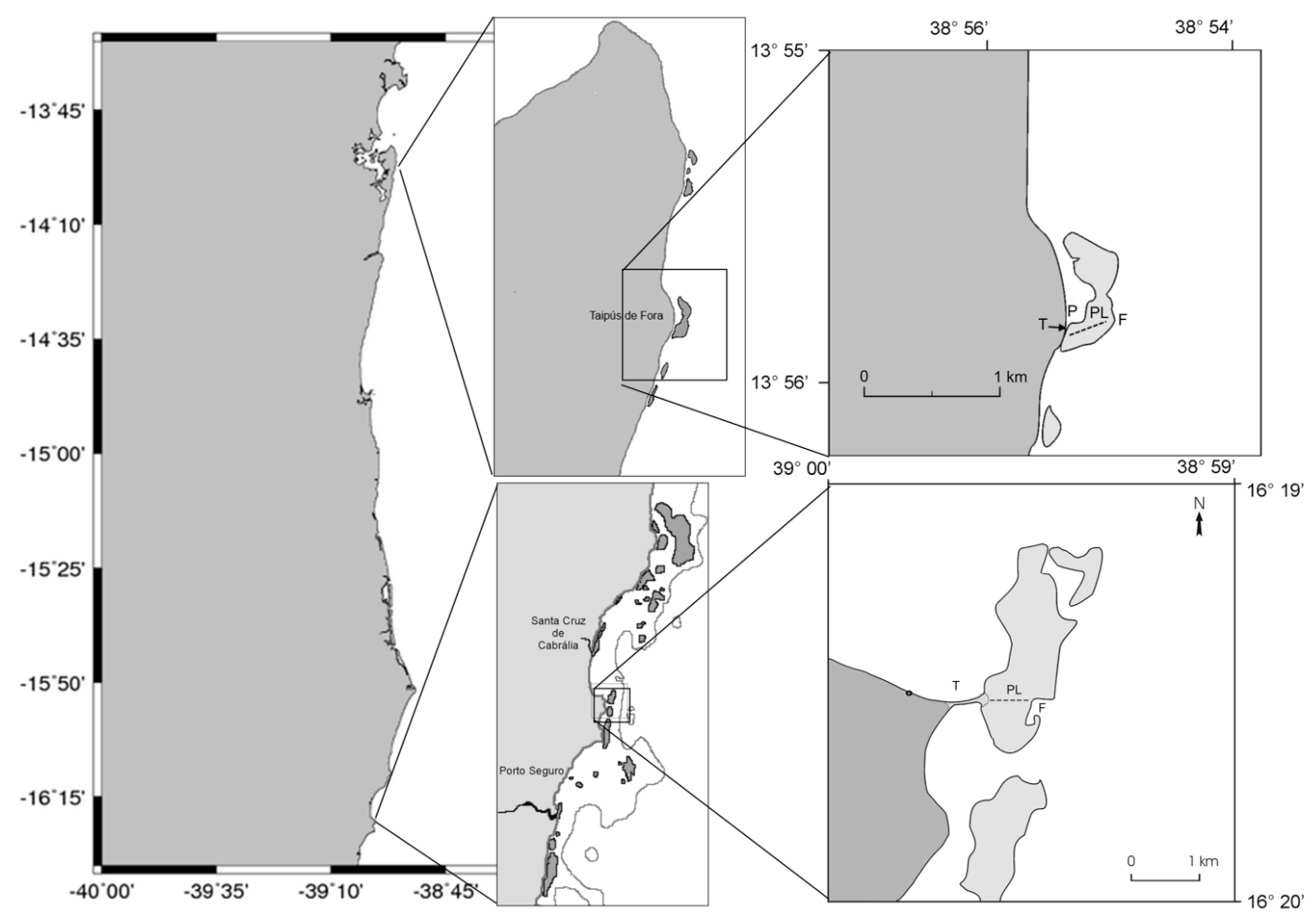

Figura 1 - Localização dos recifes de Coroa Vermelha e Taipus de Fora e pontos de coleta; $P=$ praia, $T$ = tômbolo, $P L$ e linha tracejada = transecto no platô recifal, $\mathrm{F}$ = frente recifal. 
Os dois recifes são parte de uma estrutura descontínua paralela à costa, crescendo sobre um cordão de arenito de praia (Leão, 1994). O platô recifal é exposto durante as marés baixas, formando poças rasas colonizadas por organismos bênticos. Em Coroa Vermelha o recife apresentava em média $30 \%$ de cobertura de coral $(5-45 \%)$, exclusivamente com as espécies Siderastrea stellata (Verril, 1868) e Favia gravida (Verril, 1968). S. stellata contribui com cerca de $70 \%$ desta cobertura (Santana, 2006). Não foram encontradas na literatura informações sobre a cobertura de corais em Taipus de Fora, mas as mesmas espécies estão presentes no platô deste recife. As poças de maré formadas durante a maré baixa funcionam como incubações in situ, permitindo o cálculo das taxas metabólicas bênticas a partir da variação nas concentrações de carbono inorgânico dissolvido e alcalinidade total na água.

O recife de Coroa Vermelha fica conectado com a praia durante a maré baixa por um tômbolo. É visível a presença de saídas de esgoto ao norte e ao sul deste tômbolo. Além destas fontes, o recife recebe a entrada de nutrientes e matéria orgânica através da drenagem subterrânea e de um pequeno rio poluído ao norte, o qual recebe esgoto doméstico sem tratamento das áreas urbanas adjacentes. Nas últimas três décadas o atrativo turístico tem promovido o crescimento da economia e da atividade de construção civil no litoral de Porto Seguro e Santa Cruz de Cabrália. O distrito de Coroa Vermelha apresenta grande urbanização, com vários hotéis e pousadas, além de residências. A orla é completamente ocupada por restaurantes. Devido à facilidade de acesso pelo tômbolo o recife de Coroa Vermelha tem sido impactado pelo trânsito de turistas, remoção de organismos. O platô sofre assoreamento esporádico devido ao transporte de areia pelas correntes costeiras, a qual possivelmente foi remobilizada após as obras de urbanização realizadas ao longo da costa em 2000.

O platô do recife de Taipus de Fora é conectado diretamente à praia. $\mathrm{Na}$ época da coleta, ainda não havia sido tão atingido pela atividade turística. $\mathrm{O}$ povoado de Taipus de Fora começou a surgir há 20 anos, em uma área de fazendas de coco, com o surgimento das primeiras moradias de veraneio (Aragão et al., 2010). Em 2004 ainda não havia urbanização, e poucas pousadas de pequeno porte e moradias de veraneio. Apenas dois pequenos restaurantes na praia em frente ao recife atendiam ao fluxo turístico incipiente. Esta situação modificou-se bastante desde então, tendo sido registrados 2.318 turistas na praia (12.000 m2) em momento de pico (De Souza Filho et al., 2011).

\section{Amostragem e análises químicas}

Foram realizadas duas campanhas de amostragem nos recifes de Taipus de Fora (Novembro de 2004) e Coroa Vermelha (Dezembro de 2004). Durante dois dias foram realizadas em cada local medições in situ e coleta de amostras de água na praia ou próximo ao tômbolo, na água retida em poças ao longo de um transecto no platô até a crista externa do recife, e na frente recifal (Fig. 1). Pontos de amostragem foram escolhidos aleatoriamente em poças em intervalos de 10 metros ao longo do transecto, totalizando 10 (TF) e 13 pontos (CV). Esta amostragem foi realizada duas vezes, a primeira logo após a emersão do recife e a segunda pouco antes da submersão. A coleta e medição de variáveis foi realizada a meia altura da coluna d'água, dada a variabilidade da profundidade das poças $(\sim 30$ a $50 \mathrm{~cm}$ ). Foram medidas a salinidade, temperatura e o $\mathrm{pH}$ (escala NIST), e amostras de água foram coletadas para análise de nutrientes e alcalinidade total. As amostras (cerca de $1 \mathrm{~L}$ ) foram filtradas através de filtros de fibra de vidro sinterizado tipo GF/C previamente calcinados a $450^{\circ} \mathrm{C}$ e pesados, para determinação do total de sólidos em suspensão (TSS) por gravimetria, clorofila-a (Parsons et al., 1984) e nutrientes inorgânicos dissolvidos na água filtrada (Grasshoff et al., 1983). A alcalinidade total foi analisada através de titulação com $\mathrm{HCl} 0,01 \mathrm{M}$ (Carmouze, 1994). Os dados de pH e alcalinidade total foram utilizados para o cálculo da concentração de carbono inorgânico dissolvido (CID), pressão parcial de dióxido de carbono $\left(p \mathrm{CO}_{2}\right)$ e o estado de saturação da aragonita $\left(\Omega_{\text {arag. }}\right)$ com o programa CO2SYS.EXE (Lewis \& Wallace, 1998).

\section{Fluxos de dióxido de carbono}

O fluxo de dióxido de carbono através da interface água/atmosfera foi calculado através do modelo do filme estagnante (Frankignoulle \& Gattuso, 1993) de acordo com a equação:

$$
\mathrm{F}=\mathrm{Kw} \times \alpha \times \Delta p \mathrm{CO}_{2}
$$

Onde $\mathrm{Kw}=$ coeficiente de troca com a atmosfera (velocidade de pistão; Liss \& Merlivat, 1986); m. $\mathrm{s}^{-1}$, $\alpha=$ solubilidade do $\mathrm{CO}_{2}\left(\mathrm{~mol} . \mathrm{atm}^{-1} \cdot \mathrm{m}^{-3}\right)$ e $\Delta p \mathrm{CO}_{2}$ = gradiente da pressão parcial entre a água e a atmosfera. A velocidade média do vento no período foi obtida no CPTEC/INPE (www.cptec.inpe.br). A pressão parcial atmosférica do dióxido de carbono utilizada nos cálculos (365 $\mu \mathrm{atm}$ ) foi obtida no Globalview-CO2 (www.esrl.noaa.gov/gmd/ccgg/globalview/co2). 
Calcificação-dissolução de carbonato e metabolismo líquido do ecossistema

As diversas técnicas de determinação destas taxas, suas aplicações e restrições estão descritas em Langdon et al. (2011) e Engel et al. (2011). As taxas líquidas de calcificação/dissolução de calcário foram medidas através da técnica da anomalia da alcalinidade (Smith \& Kinsey, 1978):

$$
\text { Calc. }- \text { Diss. }=\Delta \text { Alc. Total } / 2
$$

O metabolismo líquido do ecossistema (MLE = Produção primária bruta - respiração) foi calculado através da variação da média da concentração de CID obtida em cada percurso do transecto em um tempo inicial e final (Smith \& Kinsey, 1978):

$$
\mathrm{MLE}=[(\Delta \mathrm{CID} / \Delta \mathrm{t})-\text { Calc.-Diss. }]
$$

Onde MLE é o metabolismo líquido do ecossistema ( $\left.\mu \mathrm{mol} . \mathrm{L}^{-1} \cdot \mathrm{h}^{-1}\right)$, e CID é a concentração de carbono inorgânico dissolvido $\left(\mu \mathrm{mol} . \mathrm{L}^{-1}\right)$, $\mathrm{t}$ representa o intervalo em horas; Calc.-Diss. é a precipitação-dissolução de carbonato $\left(\mu \mathrm{mol} . \mathrm{L}^{-1} \cdot \mathrm{h}^{-1}\right)$.

Estas taxas não foram expressas por metro quadrado como os fluxos de $\mathrm{CO}_{2}$ uma vez que não são disponíveis as profundidades médias e rugosidade das poças para Taipus de Fora. As taxas de troca com a atmosfera não foram incluídas nos cálculos devido à sua magnitude menor e ao pequeno intervalo de tempo transcorrido entre cada transecto. Por convenção, as taxas positivas representam $\mathrm{P}>\mathrm{R}$ e calcificação > dissolução.

Em uma segunda abordagem, no dia seguinte à amostragem ao longo dos transectos foram marcadas três poças identificadas ao longo do platô recifal, nas quais foram realizadas medições no início da emersão e logo antes da submersão. O fluxo de $\mathrm{CO}_{2}$ através da interface água-atmosfera e as taxas metabólicas e de calcificação líquidas também foram determinadas utilizando as mesmas técnicas descritas acima.

As taxas calculadas referem-se aos períodos entre 9:30 e 11:30 horas (CV, transecto), 9:30 e 10:40 horas (CV, poças incubadas), 10:20 e 13:00 horas (TF, tramsecto) e 10:00 e 13:20 horas (TF, poças incubadas).

\section{Tratamento estatístico}

Foram calculadas as médias, medianas, valores máximos, mínimos e desvio padrão de todas as variáveis mensuradas, utilizando-se todas as as amostras coletadas em cada ambiente recifal (CV e TF). Dado o pequeno número de amostras e diferente entre as duas áreas, as diferenças entre a distribuição destas variáveis nos dois recifes foi avaliada através do teste $U$ de Mann-Whitney. Os valores máximos de $p$ considerados foram 0,01 e eventualmente 0,05.

\section{RESULTADOS E DISCUSSÃO}

\section{Variáveis físicas e químicas, nutrientes e clorofila}

A salinidade foi menor nos pontos de coleta junto ao tômbolo nos dois recifes do que no platô, refletindo a influência do aporte pelo lençol freático, maior em $\mathrm{CV}$, e das pequenas drenagens próximas ao recife (Tab. 1). O aumento da temperatura da água durante o período de emersão $\left(\mathrm{CV}=+7^{\circ} \mathrm{C}\right.$; $\mathrm{TF}=+12^{\circ} \mathrm{C}$ ) diminui a constante de solubilidade do $\mathrm{CaCO}_{3}$, e pode favorecer os processos de calcificação pelo aumento da estabilidade termodinâmica dos minerais carbonáticos (Gattuso et al., 1998; Howe \&

Tabela 1. Características físico-químicas da água nos recifes de Coroa Vermelha e Taipus de Fora. Média \pm desvio padrão, mínimo e máximo entre parênteses; < I.d. = inferior ao limite de detecção;

\begin{tabular}{|c|c|c|c|c|c|}
\hline & & \multicolumn{3}{|c|}{ Coroa Vermelha } & \multirow[t]{2}{*}{ Taipus de Fora } \\
\hline & & $\mathrm{N}$ & & $\mathrm{N}$ & \\
\hline Salinidade & & 25 & $\begin{array}{c}35,5 \pm 0,6 \\
(34,1-36,7)\end{array}$ & 21 & $\begin{array}{c}35,8 \pm 0,4 \\
(35,4-37,5)\end{array}$ \\
\hline Temperatura & & 22 & $\begin{array}{c}31,2 \pm 1,6 \\
(26,7-34,2)\end{array}$ & 21 & $\begin{array}{c}29,5 \pm 3,5 \\
(20,5-32,3)\end{array}$ \\
\hline Fosfato & $\mu \mathrm{M}$ & 22 & $\begin{array}{l}0,22 \pm 0,24 b \\
(<1 . d .-0.69)\end{array}$ & 21 & $\begin{array}{l}0,24 \pm 0,46 b \\
(<\text { l.d. }-1,77)\end{array}$ \\
\hline Nitrato & $\mu \mathrm{M}$ & 22 & $\begin{array}{l}0,99 \pm 0,94 b \\
(<1 . d .-2,61)\end{array}$ & 21 & $\begin{array}{l}0,30 \pm 0,39 b \\
(<1 . d .-1,14)\end{array}$ \\
\hline Nitrito & $\mu \mathrm{M}$ & 22 & $\begin{array}{l}0,22 \pm 0,38 \mathrm{a} \\
(<1 . d .-1,40)\end{array}$ & 21 & $\begin{array}{c}0,21 \pm 0,06 \mathrm{a} \\
(0,14-0,34)\end{array}$ \\
\hline $\mathrm{N}$ amoniacal & $\mu \mathrm{M}$ & 22 & $\begin{array}{l}2,62 \pm 2,51 a \\
(<\text { l.d. }-7,50)\end{array}$ & 21 & $\begin{array}{l}0,79 \pm 0,92^{a} \\
(<l . d .-2.89)\end{array}$ \\
\hline $\mathrm{pH}$ & & 25 & $\begin{array}{c}8,49 \pm 0,15 \\
(8,08-8,73)\end{array}$ & 19 & $\begin{array}{c}8,45 \pm 0,20 \\
(8,14-8,84)\end{array}$ \\
\hline $\begin{array}{l}\text { Alcalinidade } \\
\text { total }\end{array}$ & $\mu \mathrm{M}$ & 25 & $\begin{array}{c}2.094 \pm 138 \mathrm{a} \\
(1.877-2.466)\end{array}$ & 19 & $\begin{array}{r}2.226 \pm 97,8^{\mathrm{a}} \\
(1.973-2.380)\end{array}$ \\
\hline CID & $\mu \mathrm{M}$ & 25 & $\begin{array}{c}1.549 \pm 197 \mathrm{~b} \\
(1.257-1.971)\end{array}$ & 19 & $\begin{array}{c}1.674 \pm 220^{\mathrm{b}} \\
(1.344-2.089)\end{array}$ \\
\hline$p \mathrm{CO}_{2}$ & $\mu$ atm & 25 & $\begin{array}{r}160,7 \pm 113,8 \\
(55,7-508,2)\end{array}$ & 19 & $\begin{array}{l}178,1 \pm 104,5 \\
(42,2-394,5)\end{array}$ \\
\hline$\Omega$ aragonita & & 25 & $\begin{array}{c}5,8 \pm 1,1 \\
(2.9-7,4)\end{array}$ & 19 & $\begin{array}{c}5,9 \pm 1,6 \\
(3,3-9,0)\end{array}$ \\
\hline Clorofila-a & $\mu \mathrm{g}_{\dot{\sim}} \mathrm{L}^{-1}$ & 8 & $\begin{array}{c}0,53 \pm 0,89 \\
(<1 . d .-2,69)\end{array}$ & 8 & $\begin{array}{c}0,24 \pm 0,18 \\
(<1 . d .-0,45)\end{array}$ \\
\hline TSS & $\mathrm{mg}_{\mathrm{x}} \mathrm{L}^{-1}$ & 9 & $\begin{array}{c}32,6 \pm 44,5^{a} \\
(2,9-129)\end{array}$ & 8 & $\begin{array}{c}3,3 \pm 5,1^{a} \\
(<\text { I.d. }-15,4)\end{array}$ \\
\hline
\end{tabular}
sobrescrito indica nível de significância, ${ }^{a}=p \leq 0,01 ;{ }^{b}=p<0,05$.

Marshall, 2002). Isto se reflete no aumento do estado de saturação da aragonita $\left(\Omega_{\text {aragonita }}\right)$.

As concentrações de nutrientes foram significativamente menores no recife de Taipus de Fora que em Coroa Vermelha $(p<0,05)$. As concentrações de fosfato e nitrito+nitrato $\left(\mathrm{NO}_{\mathrm{x}}\right)$ registradas em CV foram menores que as encontradas por Costa Jr. et al. (2006) neste recife $\left(\mathrm{NO}_{x}, 1,7\right.$ a 3,6 $\mu \mathrm{M}$, média $\approx$ $2,9 \mu \mathrm{M}$; fosfato, 0,5 a 0,9 $\mu \mathrm{M}$, média $\approx 0,8 \mu \mathrm{M}$ ). As concentrações de nutrientes em CV são mais elevadas nas proximidades do tômbolo e menores na borda externa, sugerindo que estes estejam associados à entrada pelo lençol freático (Costa Jr. et al., 2000, 2006) e pequenas drenagens que recebem esgoto in natura. No recife de Taipus de Fora as concentrações destes nutrientes foram semelhantes às observadas 
por Costa Jr. et al. (2006) no Recife de Fora (Porto Seguro), próximo à Coroa Vermelha, porém afastado da costa. Estes são indicativos de que o recife de Taipus de Fora se encontrava menos sujeito à nutrificação que Coroa Vermelha.

Embora as maiores concentrações de clorofila-a tenham sido observadas em CV (máximo 2,7 $\mu \mathrm{g}$. $\left.\mathrm{L}^{-1}\right)$, não houve diferença significativa desta variável entre os dois recifes $(p>>0,05)$. Este valor máximo foi observado em apenas uma amostra coletada próximo ao tombolo. Esta concentração é comparável às observadas em estuários, podendo ser considerada um outlier (> valor médio + 2 D.P.).

Desconsiderando-se esta amostra, as concentrações de clorofila-a medidas nos dois recifes foram semelhantes às registradas por Costa Jr. et al. (2006) no Recife de Fora $\left(0,26\right.$ a $0,38 \mu \mathrm{g} \cdot \mathrm{L}^{-1}$; média $\approx$ $\left.0,3 \mu \mathrm{g} \cdot \mathrm{L}^{-1}\right)$, e outros recifes não impactados como em Barbados, Caribe, média igual a 0,42 $\mu \mathrm{g}$. $\mathrm{L}^{-1}$ (Tomascik \& Sander, 1985), e o recife Davis, na Grande Barreira de Corais, com médias iguais a 0,32 e 0,57 $\mu \mathrm{g} . \mathrm{L}^{-1}$ na frente externa e interna, respectivamente (Furnas et al., 1990). Estes resultados demonstram que a biomassa fitoplanctônica nas amostras não responde à concentração de nutrientes nesta escala temporal, talvez em resposta à rápida renovação da água sobre os recifes. A concentração de TSS foi superior no recife de CV $(p<0,01)$, também em grande parte devido à ressuspensão nas proximidades do tômbolo.

\section{Variáveis do sistema carbonato e fluxos de $\mathrm{CO}_{2}$}

Não houve diferença significativa do $\mathrm{pH}$ entre os dois recifes ( $p>>0,05)$, mas foi observada uma variação espacial, com valores mais altos no platô recifal

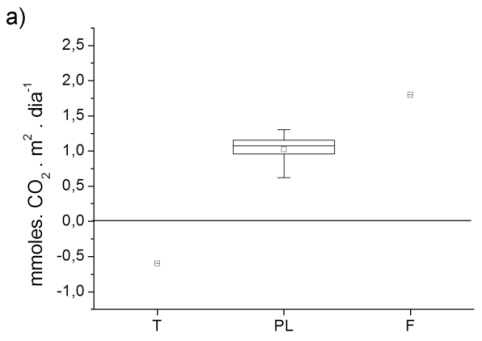

b)

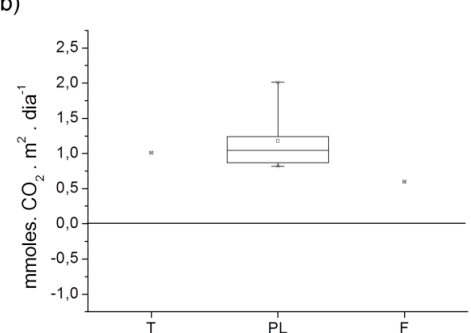

que nas amostras da borda externa, e principalmente próximas ao tômbolo e à praia.

A alcalinidade total e o CID foram significativamente maiores em TF que em CV $(p<$ 0,01 e $p<0,05$, respectivamente), e mais altas na água adjacente aos dois recifes que nas poças. $O$ predomínio de metabolismo líquido heterotrófico poderia explicar as maiores concentrações de CID, mas não da alcalinidade total. Como os valores máximos são próximos aos da Água Tropical do Atlântico Sul nesta latitude (alcalinidade $\approx 2.425 \mu \mathrm{M}$, $\mathrm{TCO}_{2} \approx 2.075 \mu \mathrm{M}$; Millero, 2006), esta diferença é um indicativo de uma maior influência da água marinha frente aos processos biogeoquímicos recifais em TF (taxas metabólicas líquidas mais próximas de zero em TF do que em CV).

Nos dois recifes a $p \mathrm{CO}_{2}$ foi bem inferior à atmosférica, indicando o papel da elevada produção primária destes ecossistemas consumindo carbono inorgânico dissolvido (Gattuso et al.,1993; Kraines et al., 1997). Os únicos pontos em que a $\mathrm{pCO}_{2}$ foi maior que a atmosférica foram aqueles junto aos tômbolos. Neste local a influência da entrada de matéria orgânica através de esgoto e da água subterrânea é maior, e sua remineralização aumenta a concentração de $\mathrm{CID}$ e diminui o $\mathrm{pH}$, resultando em uma maior $p \mathrm{CO}_{2}$. Predominaram fluxos de $\mathrm{CO}_{2}$ da atmosfera para a água nos dois recifes (Fig. 2a e 2c). Observou-se evasão de $\mathrm{CO} 2$ da água apenas nas águas adjacentes aos tômbolos (CV -0,6 e TF= -0,1 mmoles C . $\mathrm{m}^{-2}$. $\left.\mathrm{d}^{-1}\right)$. Nos experimentos em poças marcadas, os fluxos de dióxido de carbono nos dois recifes foram sempre no sentido da água (Fig. 2b e 2d). O papel de recifes costeiros como acumuladores de $\mathrm{CO}_{2}$ na maré baixa

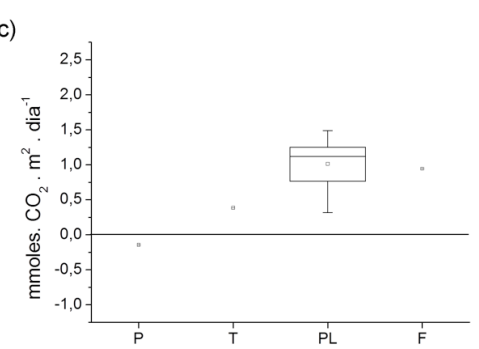

d)

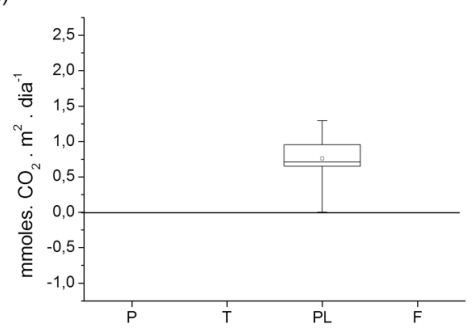

Figura 2 - Fluxos de dióxido de carbono através da interface água/atmosfera no recife de Coroa Vermelha em a) 11/12/2004, b) 12/12/2004, e Taipus de Fora,em c) 15/11/2004 e d) 16/11/2004. O box plot representa a distribuição ao longo do platô (a e c, CV 11/12, N = 13 e TF $15 / 11, N=10$ ) e nas medições nas 3 poças em dois tempos ( $b$ e d, CV 12/12 e TF 16/11, N = 6); T, P e F calculados para cada uma das amostras nestes locais; $\mathrm{T}$ = tômbolo, $\mathrm{P}=$ praia; $\mathrm{PL}=$ platô e $\mathrm{F}=$ frente recifal. Mediana, $25-75 \%$ (caixa) e máximo - mínimo. Valores positivos = fluxo da atmosfera para a água. 
já foi relatado anteriormente por Kraines et al. (1997) em um recife de franja em Bora Bay.

Este resultados de $\mathrm{pH}, \mathrm{CID}$ e $\mathrm{pCO}_{2}$ refletem tanto a influência do aporte continental de matéria orgânica e subsequente remineralização (menor $\mathrm{pH}$, $\mathrm{CID}$ e $\mathrm{pCO}_{2}$ maior), como a intensidade dos processos autotróficos que ocorrem sobre o platô recifal durante a emersão diurna (maior $\mathrm{pH}, \mathrm{CID}$ e $\mathrm{pCO}_{2}$ menor). Apesar desta heterogeneidade espacial, o pH e o $\Omega_{\text {aragonita }}$ foram praticamente iguais nos dois recifes, sempre superiores ao valores médios da água do mar (cerca de 8,1 e 2,9 respectivamente; Gattuso \& Hansson, 2011). Estes valores elevados de $\mathrm{pH}$ e $\Omega_{\text {aragonita, }}$ causados pela fotossíntese, estabelecem condições que são termodinamicamente mais favoráveis à calcificação (Gattuso et al., 1998; Leclercq et al., 2000; Reynaud et al., 2003). Como a biomassa fitoplanctônica não é expressiva, as macroalgas e o microfitobentos devem ser os responsáveis por estas altas taxas de produção primária.

\section{Metabolismo líquido do ecossistema e calcificação- dissolução de carbonato}

As taxas metabólicas líquidas obtidas com base na média das poças do transecto foram um pouco mais elevadas que as dos experimentos com poças marcadas (Figura 3a). Esta diferença pode refletir apenas a diferença nestas taxas em escala diária, uma vez que os experimentos foram realizados praticamente no mesmo horário, e um experimento piloto demonstrou não haver diferença significativa entre os dois métodos de cálculo (Longhini, C.M., comunicação pessoal). Todos os resultados indicaram autotrofia líquida, maior em CV que em TF, exceto por duas poças marcadas em Taipus de Fora. Apesar destes resultados é possível que estes recifes apresentem um MLE negativo (heterotrofia líquida), e consequentemente sejam uma fonte de $\mathrm{CO}_{2}$ para a atmosfera em uma escala igual ou superior a 24 horas, já que as medições foram realizadas apenas durante o dia. Este comportamento já foi observado em recifes de Bora Bay (Kraines et al., 1997), Kanehoe Bay (Shamberger et al., 2011) e Palau (Teneva et al., 2013).

Foi observado um predomínio de calcificação líquida (Fig. 3b), com taxas maiores em CV que em TF. As taxas líquidas de calcificação apresentaram correlação com o metabolismo (Fig. 4). As altas taxas de assimilação fotossintética de carbono inorgânico promovem um aumento do estado de saturação da aragonita e da calcita, uma vez que o maior $\mathrm{pH}$ resultante leva a uma maior disponibilidade de íons carbonato e ao processo de calcificação (Gattuso et al., 1998). A calcificação e fotossíntese durante a maré baixa diminuem a concentração de CID e a alcalinidade total. Esta intensificação do efeito do
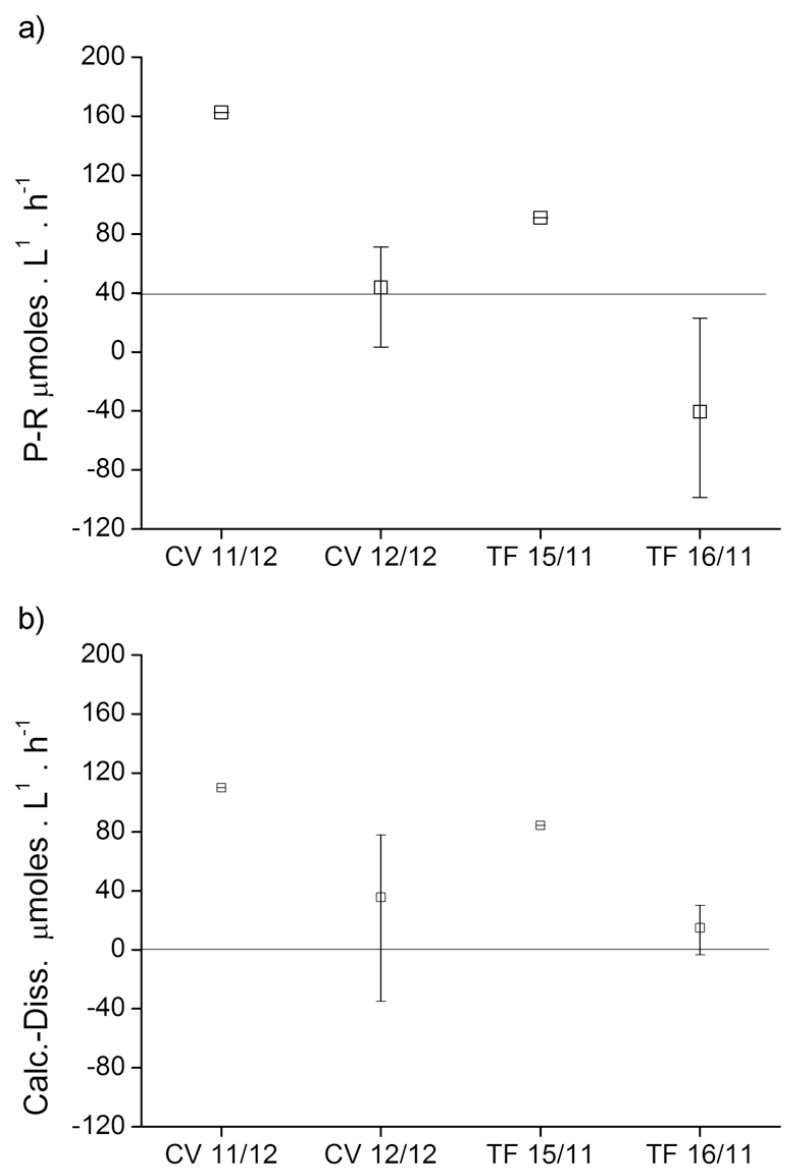

Figura 3 - a) Metabolismo líquido (P-R) e b) taxas de calcificação/ dissolução de carbonato líquidas (Calc-Diss.), em Coroa Vermelha (CV) e Taipus de Fora (TF). Mediana, máximo e mínimo. Valor de CV 11/12 e TF 15/11 calculado com base nos valores médios dos transectos nos tempos inicial e final $(\mathrm{N}=13$ e 10 respectivamente); CV 12/12 e TF 16/11 calculado com base na variação da concentração em três poças individuais $(\mathrm{N}=3)$. Valores positivos = autotrofia e calcificação líquida.

metabolismo recifal sobre o sistema $\mathrm{CO}_{2}-\mathrm{H}_{2} \mathrm{O}$ foi comparável ao observado por Kraines et al. (1997) e Manzello (2010). Os fluxos de dióxido de carbono através da interface água/atmosfera, mais lentos, não são capazes de reverter este processo através da dissolução de $\mathrm{CO}_{2}$ atmosférico na água, e as características físico-químicas da água só tendem a retornar à média marinha ao longo do período de submersão do recife.

Considerando-se as duas estratégias de determinação da MLE e calcificação-dissolução de carbonato, o cálculo com base na variação média da concentração nas poças do transecto ( $\mathrm{CV}, \mathrm{N}=13$; $\mathrm{TF}, \mathrm{N}=10$ ) apresentou valores mais elevados que os utilizando a variação da concentração em poças individuais $(\mathrm{N}=3)$. Além de diferenças diárias nestas taxas, este resultado sugere que o número de poças da segunda abordagem seja insuficiente para ser representativo de todo o platô. Porém esta permite 


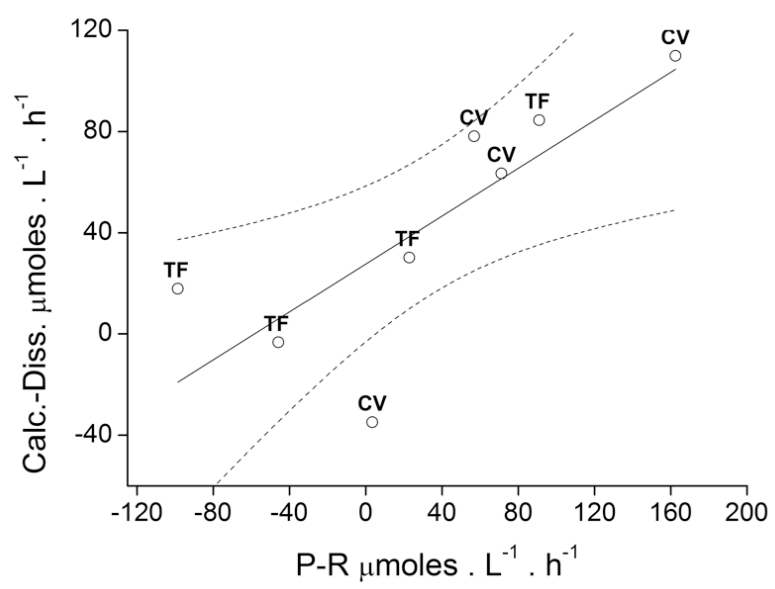

Figura 4 - Diagrama de dispersão das taxas de calcificação/dissolução de carbonato líquidas (Calc-Diss.) em função do metabolismo líquido (P-R) em Coroa Vermelha e Taipus de Fora. Valores positivos = calcificação líquida e autotrofia líquida. Regressão linear, $\mathrm{p}<0,05, \mathrm{R} 2=0,62$. Linhas tracejadas = intervalo de confiança de 0,95. A equação da regressão linear ajustada foi [Calc.-Diss.] = 0,473 P-R + 27,635.

avaliar a grande variabilidade espacial, demonstrando que há locais mais próximos ao litoral em que ocorre heterotrofia líquida e dissolução de carbonato. A meIhor estratégia para este tipo de estudo neste ambiente seria a marcação individual de todas as poças ao longo do transecto.

\section{CONCLUSÃO}

A concentração de nutrientes, especialmente o nitrogênio amoniacal, o pH, $\mathrm{CID}$ e a $\mathrm{pCO}_{2}$ indicam a influência do aporte terrestre, através da entrada direta de esgoto doméstico e água subterrânea no sistema recifal. Estas variáveis sugerem que esta entrada foi maior no recife de Coroa Vermelha que em Taipus de Fora.

A biomassa fitoplanctônica é comparativamente baixa apesar da entrada de nutrientes. A diminuição da $\mathrm{pCO}_{2}$ e aumento do $\mathrm{pH}$ nas poças do platô recifal é resultado de intensa produção primária, provavelmente por macroalgas e o microfitobentos. Esta produção primária estabelece condições termodinâmicas favoráveis aos organismos calcificadores, e gera um gradiente entre a pressão parcial de $\mathrm{CO} 2$ atmosférica e da água, com consequente fluxo para a água durante o dia.

Os dois recifes apresentaram um predomínio de metabolismo líquido do ecossistema e calcificaçãodissolução positivos (autotrofia e calcificação líquida) durante o dia. A fotossíntese controla a $\mathrm{pCO}_{2}$ e a $\Omega_{\text {aragonita }}$ durante o dia, criando condições favoráveis à calcificação. Estas taxas foram maiores em Coroa Vermelha que em Taipus de Fora, possivelmente alimentadas pela maior disponibilidade de nutrientes. Também foram observados heterotrofia e dissolução líquida de carbonatos em poças e água próximos à praia. Em uma escala temporal maior que 24 horas estes recifes podem ter heterotrofia e dissolução líquida de carbonato.

\section{AGRADECIMENTOS}

Os autores agradecem ao CNPq (Edital Universal 19/2004, proc. $\left.n^{\circ} 475552 / 2004-0\right)$, e à PROPP/UESC o apoio recebido. A segunda autora recebeu bolsa de mestrado da FAPESB. Agradecemos também a três revisores anônimos, os quais contribuíram bastante para a melhoria do trabalho.

\section{REFERÊNCIAS}

Aragão, I.R.; Abadia, B.F. \& Tupinambá, K. 2010. Paradoxos entre Taipu de Fora e Taipu de Dentro/ APA de Maraú-Bahia: Turismo e identidade local. Rosa dos Ventos, 2 (2): 80-88.

Buddemeyer, R.W.; Smith, S.V. 1999. Coral adaptation and acclimatization: A most ingenious paradox. Am. Zool. 39 (1): 1-9.

Carmouze, J-P. 1994. O Metabolismo dos Ecossistemas Aquáticos. Fundamentos teóricos, métodos de estudo e análises químicas. Ed. Edgard Blücher/ FAPESP, São Paulo, 253 p.

Costa Jr., O.S.; Leão, Z.M.A.N.; Nimmo N. \& Attrill, M.J. 2000. Nutrification impacts on coral reefs from northern Bahia, Brazil. Hydrobiol. 440:307-315.

Costa Jr., O.S.; Attrill, M.J.\& Nimmo, M. 2006 Seasonal and spatial controls on the delivery of excess nutrients to nearshore and offshore coral reefs of Brazil. J. Mar. Syst. 60:63-74.

De Souza Filho, J.R.; Silva, I.R \& Ferreira, D.F., 2011. Socio-Environment Analysis as a Tool for Coastal Management: the Case of Maraú Peninsula, Bahia, Brazil. In: Micallef, A. (ed.), MCRR3-2010 Conference Proceedings, Journal of Coastal Research, Special Issue, No. 61, pp. 446-451. Grosseto, Tuscany, Italy, ISSN 0749-0208.

Engel, A.; Barcelos e Ramos, J.; Geider, R.; Hutchins, D.A.; Lee, C.; Rost, B.; Röttgers, R. \& Thingstad, F. 2011. Production and export of organic matter. In: Riesebell, U., Fabry, V.J.; Hansson, L \& Gattuso, J-P. (eds.) Guide to best practices for ocean acidification research and data reporting. Publications Office of the European Union, Luxembourg, p. 181-200.

Falter J.L.; Lowe, R.J.; Zhang, Z. \& McCulloch M. 2013. Physical and biological controls on 
the carbonate chemistry of coral reef waters: Effects of metabolism, wave forcing, sea level, and Geomorphology. PLoS One 8(1), e53303, doi:10.1371/journal.pone.0053303

Frankignoulle, M. \& Gattuso, J.P. 1993. Air-sea CO2 exchange in coastal systems. In: Wollanski, R.; Mackenzie, F.T. \& Chou, L. (eds.) Interactions of $\mathrm{C}, \mathrm{N}, \mathrm{P}$ and $\mathrm{S}$ biogeochemical cycles and global change. NATO ASI Series. Springer-Verlag, Berlin, pp 233-248.

Frankignoulle, M.; Canon, C. \& Gattuso, J-P. 1994 Marine calcification as a source of carbon dioxide: positive feedback of increasing atmospheric $\mathrm{CO} 2$. Limnol. Oceanogr. 39:458-462.

Furnas, M.J.; Mitchell, A.W.; Gilmartin, M. \& Revelante, N. 1990. Phytoplankton biomass and primary production in semi-enclosed reef lagoons of the central Great Barrier Reef, Australia. Coral Reefs 9, 1-10.

Gattuso, J-P.; Pichon, M.; Delesalle, B. \& Frankignoulle, M. 1993. Community metabolism and air-sea CO2 fluxes in a coral reef (Moorea, French Polynesia). Mar. Ecol. Prog. Ser. 96:259-267.

Gattuso, J.P.; Frankignoulle, M.; Bourge, I.; Romaine, S. \& Buddemeier, R. W. 1998. Effect of Calcium Carbonate Saturation of Seawater on Coral Calcification. Glob. Plan. Change 18:37-46.

Gattuso, J.P.; Frankignoulle, M. \& Smith, S. 1999. Measurement of Community Metabolism and Significance in the Coral Reef CO2 SourceSink Debate. Proc. Natle. Acad. Sci. USA 23:13017-13022.

Gattuso, J.P. \& Hansson, L. 2011. Ocean acidification: Background and history. In: Gattuso, J.P. \& Hansson, L. (eds.) Ocean Acidification. Oxford University Press, Oxford, pp $1-17$.

Grasshoff, K.; Ehrardt, M. \& Kremling, K. 1983. Methods of Seawater Analysis. Verlag Chemie, Weinhein, $419 p$.

Howe, S. A\& Marshall, A. T. 2002. Temperature effects on calcification rate and skeletal deposition in the temperate coral, Plesiastrea versipora (Lamarck). Jour. Exp. Mar. Biol. Ecol. 275: 63-81.

Kraines, S.B.; Suzuki, Y.; Omor, I T.; Shitashima ,K.; Kanahara,S. \& Komiyamah, H. 1997. Carbonate dynamics of the coral reef system at Bora Bay, Miyako Island. Mar. Ecol. Prog. Ser. 156:1 -16.

Langdon, C.; Gattuso, J-P. \& Andersson, A. 2011. Measurements of calcification and dissolution of benthic organisms and communities. In: Riesebell, U., Fabry, V.J.; Hansson, L \& Gattuso, J-P. (eds.) Guide to best practices for ocean acidification research and data reporting. Publications Office of the European Union, Luxembourg, p. 213-232.
Leão, Z.M.A.N. 1994. The coral reefs of Southern Bahia. In: Hetzel B, Castro CB (eds.) Coral of Southern Bahia. Nova fronteira, Rio de Janeiro, $159 \mathrm{p}$.

Leclercq, N.; Gattuso, J-P. \& Jaubert, J. 2000. CO2 partial pressure controls de calcification rate of a coral community. Global Change Biol 6:329-334.

Leclercq, N. \& Gattuso, J.P. 2002. Primary Production, Respiration, and Calcification of a Coral Reef Mesocosm under Increased CO2 Partial Pressure. Limnol. Oceanogr. 47(2):558-564.

Lewis, E. \& Wallace, D.W.R. 1998. Program Developed for CO2 System Calculations.ORNL/CDIAC-105. Carbon Dioxide Information Analysis Center, Oak Ridge National Laboratory, U.S. Department of Energy, Oak Ridge,TN.

Liss, P.S. \& Merlivat. L. 1986. Air-sea gas exchange rates: introduction and synthesis. In: BuartMenard, P. (ed.) The role of air-sea exchange in geochemical cycling. D. Reidel Publishing Company, Utrecht, pp 113-128.

Manzello, D.P. 2010. Ocean acidification hotspots: Spatiotemporal dynamics of the seawater $\mathrm{CO} 2$ system of eastern Pacific coral reefs. Limnol. Oceanogr. 55:239-248.

Millero, F.J. 2006. Chemical Oceanography. 3rd ed. CRC Press, Boca Raton, $496 \mathrm{p}$.

Orr, J.C.; Fabry, V.J.; Aumont, O.; Bopp, L.; Doney, S.C.; Feely, R.A.; Gnanadesikan, A.; Gruber, N.; Ishida, A.; Joos, F.; Key, R.M.; Lindsay, K.; MaierReimer, E.; Matear, R.; Monfray, P.; Mouchet, A.; Najjar, R.G.; Plattner, G-K.; Rodgers, K.B.; Sabine, C.L.; Sarmiento, J.L.; Schlitzer, R.; Slater, R.D.; Totterdell, I.J.; Weirigi, M-F.; Yamanaka, Y. \& Yool, A. 2005. Anthropogenic ocean acidification over the twenty-first century and its impact on calcifying organisms. Nature 437:681-686.

Reynaud, S.; Leclercq, N.; Romaine-Lioud, S.; FerrierPagès, C.; Jaubert, J. \& Gattuso. J-P. 2003. Interacting effects of $\mathrm{CO} 2$ partial pressure and temperature on photosynthesis and calcification in a scleractinian coral. Global Change Biol. 9:1660-1668.

Santana, T.B. 2006. Caracterização hidroquímica e distribuição das assembléias macrobênticas na porção central do recife de Coroa Vermelha, Santa Cruz de Cabrália, Bahia. Tese de mestrado. Universidade Estadual de Santa Cruz. 66 p.

Shamberger, K.E.F.; Feely, R.A.; Sabine, C.L.; Atkinson, M.J.; DeCarlo, E.H.; Mackenzie, F.T.; Drupp, P.S. \& Butterfield, D.A. 2011. Calcification and organic production on a Hawaiian coral reef. Mar. Chem. 127: 64-75.

Smith, S.V. 1973. Carbon dioxide dynamics: a record of organic carbon production, respiration, and 
calcification in the Eniwetok reef flat community. Limnol. Oceanogr. 18:106-120.

Smith, S.V. 1974. Coral reef carbon dioxide flux. Proc. 2nd Int. Coral Reef Symp. 1:77-85.

Smith, S.V. 1981. The Houtman Abrolhos Islands: Carbon metabolism of coral reefs at high latitude. Limnol. Oceanogr. 26:612-621.

Smith, S.V. \& Kinsey, D.W. 1978. Calcification and organic carbon metabolism as indicated by carbon dioxide. In: Stoddart, D.R. \& Johannes, R.E. (eds) Coral Reefs: Research methods. Monogr. Oceanogr. Methodol. 5. UNESCO, pp 469-484.

Smith, S.V. 2013. Parsing the oceanic calcium carbonate cycle: a net atmos-pheric carbon dioxide source, or a sink? L\&O e-Books. Association for the Sciences of Limnology and Oceanography (ASLO) Waco, TX. 10.4319/svsmith.2013.978-0-9845591-2-1.
Teneva, L.; Dunbar, R.B.; Mucciarone, D.A.; Dunckley, J.F. \& Koseff, J.R. 2013. High-resolution carbon budgets on a Palau back-reef modulated by interactions between hydrodynamics and reef metabolism. Limnol. Oceanogr. 58(5): 1851-1870.

Tomascik, T. \& Sander, F. 1985. Effects of eutrophication on reef- building corals: I. Growth rate of the reefbuilding coral Montas- trea annularis. Mar. Biol. 87, 143-155.

Tribollet, A.; Langdon, C.; Golubic, S. \& Atkinson, M. 2006. Endolithic microflora are major primary producers in dead carbonate substrates of Hawaiian coral reefs. J. Phycol. 42:292-303.

Ware, R.W.; Smith, S.V. \& Reaka-Kudla, M.L. 1991. Coral reefs: sources or sinks of atmospheric CO2? Coral Reefs 11:127-130.

Submetido: Julho/2013

Revisado: Julho/2015

Aceito: Agosto/2015 